\title{
RELATIVE HAUPTVERMUTUNG FOR NEIGHBORHOODS OF 1-FLAT SUBMANIFOLDS WITH CODIMENSION TWO
}

\author{
BY MITSUYOSHI KATO ${ }^{1}$
}

Communicated by William Browder, April 21, 1969

1. Recently Kirby and Siebenmann have given general solutions of Hauptvermutung [5] and relative Hauptvermutung for neighborhoods of locally flat submanifolds [6]. In this note we announce some results about relative Hauptvermutung for neighborhoods of 1-flat submanifolds with codimension two (compare [11] and [3]).

We shall say that manifold pairs $(Q, M)$ and $\left(Q^{\prime}, M^{\prime}\right)$ are topologically micro-equivalent, if there are open neighborhoods $U, U^{\prime}$ of $M, M^{\prime}$ in $Q, Q^{\prime}$ and a homeomorphism $h:(U, M) \rightarrow\left(U^{\prime}, M^{\prime}\right)$, called a topological micro-equivalence between $(Q, M)$ and $\left(Q^{\prime}, M^{\prime}\right)$. We shall say that PL manifold pairs $(Q, M)$ and $\left(Q^{\prime}, M^{\prime}\right)$ are PL micro-equivalent, if there are open neighborhoods $V, V^{\prime}$ of $M, M^{\prime}$ in $Q, Q^{\prime}$ and a PL homeomorphism $g:(V, M) \rightarrow\left(V^{\prime}, M^{\prime}\right)$, called a PL micro-equivalence between $(Q, M)$ and $\left(Q^{\prime}, M^{\prime}\right)$.

We shall prove the following

Theorem A. Let $(Q, M)$ and $\left(Q^{\prime}, M^{\prime}\right)$ be proper $P L$ orientable $(4,2)$ manifold pairs. Suppose that $M$ is compact and that there is a topological micro-equivalence $h:(U, M) \rightarrow\left(U^{\prime}, M^{\prime}\right)$. Then there is a $P L$ microequivalence $g:(V, M) \rightarrow\left(V^{\prime}, M^{\prime}\right)$. Further, if $h \mid M$ is already $P L$, then we can take $g$ so that $\mathrm{g}|M=h| M$.

In order to extend this result to the higher dimensional case, we need some niceness condition for singularities.

Let $(Q, M)$ be a proper PL $(m+2, m)$-manifold pair. We shall say that $M$ is locally flat at a point $x$ of $M$, if the link pair of $x$ in $(Q, M)$ is PL equivalent to the standard sphere or ball pair. If $M$ is not locally flat at $x$, then $x$ is called a singular point of $M$, and the link pair is called the singularity. We shall say that $M$ is 1 -flat in $Q$, if the set of singular points consists of isolated points. Note that if $M$ is 1 -flat in $Q$, the link pair of any point of $M$ in $(Q, M)$ is a locally flat PL $(m+1, m-1)$-sphere or ball pair; i.e. PL $(m-1)$-knot or disk knot, and that if $M$ is of dimension two, then $M$ is always 1 -flat in $Q$. We

\footnotetext{
1 Work supported by Sakkokai Foundation and National Science Foundation (GP-7952X).
} 
shall say that a PL $(m-1)$-knot or disk knot is nice, if the complement of an open regular neighborhood of the embedded sphere or ball has a fundamental group isomorphic with that of the boundary.

Theorem B. Let $(Q, M)$ and $\left(Q^{\prime}, M^{\prime}\right)$ be proper orientable $P L$ $(m+2, m)$-manifold pairs. Suppose that $M, M^{\prime}$ are compact, 1-flat in $Q, Q^{\prime}$ with nice singularities, and $m \geqq 5$. If there is a topological microequivalence $h:(U, M) \rightarrow\left(U^{\prime}, M^{\prime}\right)$ such that $h \mid M$ is $P L$, then there is a $P L$ micro-equivalence $\mathrm{g}:(V, M) \rightarrow\left(V^{\prime}, M^{\prime}\right)$ such that $\mathrm{g}|M=h| M$.

On the contrary we have the following remarkable counterexample.

THEOREM C. For each even integer $m \geqq 4$, there are abstract regular neighborhoods $(N, K),\left(N^{\prime}, K^{\prime}\right)$ of $m$-spheres $K, K^{\prime}$ with codimension two which are locally flat except for single points, resp., and an embedding $h:(N, K) \rightarrow\left(N^{\prime}, K^{\prime}\right)$ such that $h \mid K$ is $P L$, but there is no PL microequivalence between $(N, K)$ and $\left(N^{\prime}, K^{\prime}\right)$.

This example ensures that even if the local flatness of a submanifold breaks at only one point, the relative Hauptvermutung for neighborhoods is false, which should be compared with the affirmative answer for the locally flat case by Kirby and Siebenmann [6].

2. Topological invariance problem of singularities. Let $(Q, M)$ be a proper PL $(m+2, m)$-manifold pair. For a point $x$ of $M$, by a link pair of $x$ in $(Q, M)$ we shall mean a pair $(l \mathrm{k}(x, K), l \mathrm{k}(x, L))$, written $l \mathrm{k}(x ; K, L)$, of links of $x$ in $K$ and $L$ for some division $(K, L)$ (or partition) of $(Q, M)$. Then the PL homeomorphism class of the link pair $l \mathrm{k}(x ; K, L)$ does not depend on the choice of the division $(K, L)$ of $(Q, M)$ and will be denoted by $l \mathrm{k}(x ; Q, M)$. We examine the topological invariance of $l \mathrm{k}(x ; Q, M)$.

Lemma 1. Let $(Q, M)$ and $\left(Q^{\prime}, M^{\prime}\right)$ be proper $P L(m+2, m)$-manifold pairs. Suppose that there is a topological micro-equivalence $h:(U, M)$ $\rightarrow\left(U^{\prime}, M^{\prime}\right)$. Then $l \mathrm{k}(x ; Q, M)$ and $l \mathrm{k}\left(x ; Q^{\prime}, M^{\prime}\right)$ are topologically invertible cobordant.

Proof. One can take divisions $(K, L),\left(K_{1}, L_{1}\right)$ of $(Q, M)$ and $\left(K^{\prime}, L^{\prime}\right),\left(K_{1}^{\prime}, L_{1}^{\prime}\right)$ of $\left(Q^{\prime}, M^{\prime}\right)$ so that

and

$$
\begin{aligned}
c_{1} & =\operatorname{cl}\left(\operatorname{st}\left(h x ; K^{\prime}, L^{\prime}\right)-h(\operatorname{st}(x ; K, L))\right), \\
c & =\operatorname{cl}\left(h(\operatorname{st}(x ; K, L))-\operatorname{st}\left(h x ; K_{1}^{\prime}, L_{1}^{\prime}\right)\right)
\end{aligned}
$$

$$
c_{2}=\operatorname{cl}\left(\operatorname{st}\left(h x ; K_{1}{ }^{\prime}, L_{1}{ }^{\prime}\right)-h\left(\operatorname{st}\left(x ; K_{1}, L_{1}\right)\right)\right.
$$

are topological cobordisms of manifold pairs, where, for example. 
st $(x ; K, L)$ denotes the star pair (st $(x, K)$, st $(x, L))$. Since the composition $c_{1} \circ c=\operatorname{cl}\left(\mathrm{st}\left(h x ; K^{\prime}, L^{\prime}\right)-\operatorname{st}\left(h x ; K_{1}^{\prime}, L_{1}^{\prime}\right)\right)$ is PL equivalent to $l \mathrm{k}\left(h x ; K^{\prime}, L^{\prime}\right) \times[0,1]$ and $c \circ c_{2}=\operatorname{cl}\left(h\left(\mathrm{st}(x ; K, L)-\mathrm{st}\left(x ; K_{1}, L_{1}\right)\right)\right)$ is topologically equivalent to $l \mathrm{k}(x ; K, L) \times[0,1]$, it follows that $c$ is the required invertible cobordism between $1 \mathrm{k}(x ; Q, M)$ and $l \mathrm{k}\left(h x ; Q^{\prime}, M^{\prime}\right)$, completing the proof.

Let $(A, B)$ and $\left(A^{\prime}, B^{\prime}\right)$ be proper PL manifold pairs. Let $N$ and $N^{\prime}$ be derived neighborhoods of $B$ and $B^{\prime}$ in $A$ and $A^{\prime}$, resp. Putting $E=\operatorname{cl}(A-N)$ and $E^{\prime}=\operatorname{cl}\left(A^{\prime}-N^{\prime}\right)$, we shall call them exteriors of $B$ and $B^{\prime}$ in $A$ and $A^{\prime}$, resp.

Lemma 2. Suppose that $(A, B)$ and $\left(A^{\prime}, B^{\prime}\right)$ are topologically invertible cobordant. Then there is a map $f:(A, B) \rightarrow\left(A^{\prime}, B^{\prime}\right)$ such that $f(E)$ $=E^{\prime}, f(\partial E)=\partial E^{\prime}, f(N)=N^{\prime}$ and $f\left((E, \partial E):(E, \partial E) \rightarrow\left(E^{\prime}, \partial E^{\prime}\right)\right.$ is a homotopy equivalence.

In fact, by the argument of Stallings [12, Theorem 2], $(A, B) \times R$ and $\left(A^{\prime}, B^{\prime}\right) \times R$ are homeomorphic. The composition $(A, B) \stackrel{\times 0}{\longrightarrow}(A, B)$ $\times R \rightarrow\left(A^{\prime}, B^{\prime}\right) \times R \rightarrow\left(A^{\prime}, B^{\prime}\right)$ can be deformed to the desired map, (refer to [2, proof of Lemma 5.1]), where the third map is the projection onto the first factor.

Here are examples of PL knots or disk knots for which topological invertible cobordism implies PL equivalence.

EXAMPLE 1. Let $(A, B)$ be a PL m-knot (or disk knot) topologically invertible cobordant to the standard one. Suppose that $m \neq 2($ or $m \neq 2,3)$. Then $(A, B)$ is $P L$ equivalent to the standard one.

This is an immediate consequence of the unknotting theorem.

EXAMPLE 2. Let $(A, B)$ and $\left(A^{\prime}, B^{\prime}\right)$ be PL 1-knots (classical knots) (or disk knots). If $(A, B)$ and $\left(A^{\prime}, B^{\prime}\right)$ are topologically invertible cobordant, then they are $P L$ equivalent.

This follows from Waldhausen's Theorem [15]. In fact, Schoenflies Theorem and the existence of the minimal surface for a classical knot guarantees that an exterior of a classical knot is an irreducible and sufficiently large 3-manifold. By making use of Wall's surgery obstruction theory [14] (also see [9]) and the pseudo-isotopy classification theorem of PL homeomorphisms of $S^{m-1} \times S^{1}$ ([1] and [2]) we have a partial answer to the higher dimensional case:

EXAMPLE 3. Let $(A, B)$ and $\left(A^{\prime}, B^{\prime}\right)$ be nice $P L(m-1)$-knots or disk knots. Suppose that $m \geqq 5$. If $(A, B)$ and $\left(A^{\prime}, B^{\prime}\right)$ are topologically invertible cobordant, then they are $P L$ equivatent.

The following counterexample is due to Siebenmann [10, p. 741]. Counterexample (Siebenmann). For each even integer $m \geqq 4$, 
there are topologically distinct $P L(m-1)$-knots $(S, \Sigma)$ and $\left(S^{\prime}, \Sigma^{\prime}\right)$ that are $P L$ invertible cobordant.

3. Outline of the proof of theorems. In [7] Noguchi defined the Euler class $\chi(Q, M) \in H^{2}(M, Z)$ for a second derived neighborhood of a compact orientable proper PL $m$-submanifold $M$ in an orientable PL $(m+2)$-manifold $Q$. It is to be noted that if $m=2$, then by Takase [13] the Euler number $\langle\chi(Q, M),[M]\rangle$ is just the self-intersection number of the fundamental homology class $(M)$ of $M$ in $Q$; i.e. $\langle D(M),(M)\rangle$, where $[M]$ is the fundamental homology class of $M$ and $D: H_{2}(Q) \rightarrow H^{2}(Q, \partial Q)$ is the Poincare duality. If $m \geqq 3$ and if $M$ is 1-flat with singular points $x_{1}, \cdots, x_{n}$, then $i^{*} \chi(Q, M)=\chi\left(Q_{0}, M_{0}\right)$, where $Q_{0}=Q-U_{i=1}^{n} \operatorname{st}\left(x_{i}, K\right), M_{0}=M-U_{i=1}^{n} \operatorname{st}\left(x_{i}, L\right)$ for the first barycentric subdivision $(K, L)$ of a division of $(Q, M), \chi\left(Q_{0}, M_{0}\right)$ is the Euler class of the normal bundle of $M_{0}$ in $Q_{0}$ and $i^{*}: H^{2}(M, Z)$ $\rightarrow H^{2}\left(M_{0}, Z\right)$ is the monomorphism induced by the inclusion map $i: M_{0} \rightarrow M$.

Now we are ready to give an outline of the proof of theorems. Suppose that there is a topological micro-equivalence $h:(U, M)$ $\rightarrow\left(U^{\prime}, M^{\prime}\right)$ and that $m \neq 3,4$, and $h \mid M$ is already PL, if $m \geqq 5$. In case $m=2$, by Hauptvermutung for surfaces we may assume that $h \mid M$ is already PL. We will show that the PL homeomorphism $h \mid M$ can be extended to a PL micro-equivalence $g:(V, M) \rightarrow\left(V^{\prime}, M^{\prime}\right)$. For this in view of Noguchi's Theorem [8] we have only to show that $(h \mid M)^{*} \chi\left(Q^{\prime}, M^{\prime}\right)=\chi(Q, M)$ and $h \mid M$ preserves singular points so that $l \mathrm{k}\left(h x_{i} ; Q^{\prime}, M^{\prime}\right)=1 \mathrm{k}\left(x_{i} ; Q, M\right)$ for all singular points $x_{i}$. First, by Example 1, $h \mid M$ preserves singular points; i.e. $h x$ is a singular point of $M^{\prime}$ in $Q^{\prime}$ if and only if $x$ is a singular point of $M$ in $Q$ and secondly, by Examples 2 and $3 l \mathrm{k}\left(h x_{i} ; Q^{\prime}, M^{\prime}\right)=l \mathrm{k}\left(x_{i} ; Q, M\right)$ for all singular points $x_{i}$ of $M$ in $Q$. Thirdly notice that $h$ can be deformed to a map whose restriction to the locally flat part is a fiber homotopy equivalence between normal bundles for the locally flat parts $\left(Q_{0}, M_{0}\right)$ and $\left(Q_{0}^{\prime}, M_{0}^{\prime}\right)$. This together with the interpretation of $\chi(Q, M)$ given in the above shows that $(h \mid M)^{*} \chi\left(Q^{\prime}, M^{\prime}\right)=\chi(Q, M)$. Thus Noguchi's Theorem [8] completes the proof of Theorems A and B. In order to prove Theorem $C$, for each even integer $m \geqq 4$ we take PL $(m-1)$-knots $(S, \Sigma)$ and $\left(S^{\prime}, \Sigma^{\prime}\right)$ given in the counterexample above. Form PL $(m+2)$-manifolds $N=0 * S U_{\left(\Sigma \times D^{2}\right)} D^{m} \times D^{2}$ and $N^{\prime}=0 * S^{\prime} \bigcup_{\left(\Sigma^{\prime} \times D^{2}\right)} D^{m} \times D^{2}$ from cones $0 * S$ and $0^{\prime} * S^{\prime}$ by attaching handles of index $m$ along collar neighborhoods $\left(\Sigma \times D^{2}\right)$ and $\left(\Sigma^{\prime} \times D^{2}\right)$ of $\Sigma$ and $\Sigma^{\prime}$ in $S$ and $S^{\prime}$, resp. Then $N$ and $N^{\prime}$ are abstract regular neighborhoods of $K=0 * \Sigma U_{\Sigma}\left(D^{m} \times 0\right)$ and $K^{\prime}=0^{\prime} * \Sigma^{\prime} \bigcup_{\Sigma^{\prime}}\left(D^{m} \times 0\right)$ 
which have only single singularities $(S, \Sigma)$ and $\left(S^{\prime}, \Sigma^{\prime}\right)$, resp. By the invertibility of the PL cobordism between $(S, \Sigma)$ and $\left(S^{\prime}, \Sigma^{\prime}\right)$, we have a topological embedding $h:(N, K) \rightarrow\left(N^{\prime}, K^{\prime}\right)$ such that $h \mid K$ is a PL homeomorphism, (refer to $[4, \S 5]$ and $[11]$ ). Hence $(N, K)$ and $\left(N^{\prime}, K^{\prime}\right)$ are topologically micro-equivalent. On the other hand, singularities $(S, \Sigma)$ and $\left(S^{\prime}, \Sigma^{\prime}\right)$ are distinct. Therefore, $(N, K)$ and $\left(N^{\prime}, K^{\prime}\right)$ are never PL micro-equivalent, completing the outline of the proof of Theorem $\mathrm{C}$.

\section{REFERENCES}

1. W. Browder, Diffeomorphisms of 1-connected manifolds, Trans, Amer. Math. Soc. 128 (1967), 155-163.

2. M. Kato, A concordance classification of $P L$ homeomorphisms of $S^{p} \times S^{q}$, Topology (to appear).

3. - Regular neighborhoods are not topologically invariant, Bull. Amer. Math. Soc. 74 (1968), 988-991.

4. - Geometrical operations of Whitehead groups (to appear).

5. R. Kirby and L. C. Siebenmann, On the triangulations of manifolds and the Hauptvermutung, Bull. Amer. Math. Soc. 75 (1969), 742-749.

6. - A straightening theorem and a Hauptvermutung for pairs, Notices Amer. Math. Soc. 16 (1969), 582.

7. H. Noguchi, One flat 3-manifolds in 5-space, Osaka J. Math. 1 (1964), 117-125.

8. - One flat submanifolds with codimension two, Illinois J. Math. 13 (1969), 220-223.

9. R. K. Lashof and J. L. Shaneson, Classification of knots in codimension two, Bull. Amer. Math. Soc. 75 (1969), 171-175.

10. L. C. Siebenmann, On the homotopy type of compact topological manifolds, Bull. Amer. Math. Soc. 74 (1968), 738-742.

11. L. C. Siebenmann and J. Sondow, Some homeomorphic sphere pairs that are combinatorially distinct, Comment. Math. Helv. 41 (1966-67), 261-272.

12. J. Stallings, On infinite processes leading to differentiability in the complement of a point. Differential and combinatorial topology, Princeton Univ. Press, Princeton, N. J., 1965, pp. 245-254.

13. R. Takase, Note on orientable surfaces in 4-space, Proc. Japan Acad. 39 (1963), 424.

14. C. T. C. Wall, Surgery on compact manifolds, Mimeographed notes.

15. F. Waldhausen, On irreducible 3-manifolds which are sufficiently large, Ann. of Math. (2) 87 (1968), 56-88.

Tokyo Metropolitan University, Tokyo, Japan and

Institute for Advanced Study, Princeton, New Jersey 08540 